\title{
Effect of 5'-S-Methyltioadenosine on Growth and Ultrastructure of Epimastigote Forms of Trypanosoma cruzi
}

P.S. Luize*, T. Ueda-Nakamura*, A. Zimmermann*, G.J. Vidotti*, B.P. Dias Filho*, J.A. MorgadoDíaz** and C.V. Nakamura*

* Universidade Estadual de Maringá, Departamento de Análises Clínicas, Av. Colombo 5790, CEP 87020-900, Maringá, PR Brasil. cvnakamura@uem.br

** Instituto Nacional de Câncer, Divisão de Biologia Celular, Coordenação de Pesquisa, Praça Cruz Vermelha, 23, $6^{\circ}$ andar, CEP 20230-130, Rio de Janeiro, RJ Brasil.

Phytoterapy has received considerable attention in the search for alternatives to chemotherapy in parasitic diseases control. Plants still provide chemical diversity and bioactivity, which has led to the development of hundreds of pharmaceutical drugs. Pedilanthus tithymaloides (L) Poit (Euphorbiaceae), locally known as "dois amores" is used in traditional medicine as antitumoral, abortive and to treat sore throat $[1,2]$. In the Latin America, an estimated 25 million people are infected with Trypanosoma cruzi, the etiologic agent of Chagas' disease [3]. Its treatment is today still a challenge and more effective drugs are urgently needed to treat chagasic patients.

In the present study we have investigated the toxic activity and ultrastructural alterations of 5'-SMethyltioadenosine purified from leaves of Pedilanthus tithymaloides against epimastigote forms of $T$. cruzi. The plant was collected in Altonia-PR, Brazil, and a voucher specimem (HUM 6104) is deposited in the Herbarium of the Universidade Estadual de Maringá. The compound was isolated from ethanolic extract by column, contercurrent and TLC preparative chromatography. T. cruzi "Y" strain was cultivated in LIT medium containing $0.17,0.33,1.7,3.4,17 \mu \mathrm{M}$ of the compound dissolved in dimethyl sulfoxide (DMSO). Dose-response growth-curves showed major trypanosomicidal activity with an $\mathrm{IC}_{50}$ of $\sim 0.236 \mu \mathrm{M}$, after 120 hours of incubation (Figure 1).

In order to investigate the influence of 5'-S-Methyltioadenosine in protozoan's ultrastructure, cells treated with the compound were fixed in $2.5 \%$ glutaraldehyde. Postfixation was carried out in $1 \%$ osmium tetroxide, dehydrated in acetone, and samples were embedded in Epon. Ultrathin sections were observed in a Zeiss EM-900 electron microscope. Cells treated with 5'-S-Methyltioadenosine presented several morphological changes such as invaginations of the inner mitochondrial membrane, mitochondrial swelling, and Golgi complex vesiculation (Figure 2). These results indicate that 5'-SMethyltioadenosine compound have a progressive inhibitory activity on the growth of epimastigote forms of T. cruzi and determine some ultrastructural mitochondrial alterations.

\section{References}

[1] - M.P. Correa.- Dicionário das plantas úteis do Brasil e das exóticas cultivadas. vol. I Rio de Janeiro, Imprensa Nacional, 1984.

[2] - T.T. Roig.- Plantas medicinales, aromáticas o venenosas de Cuba, 1968.

[3] - World Health Organization. 1991. Control of Chagas' disease. WHO 811:1-93 


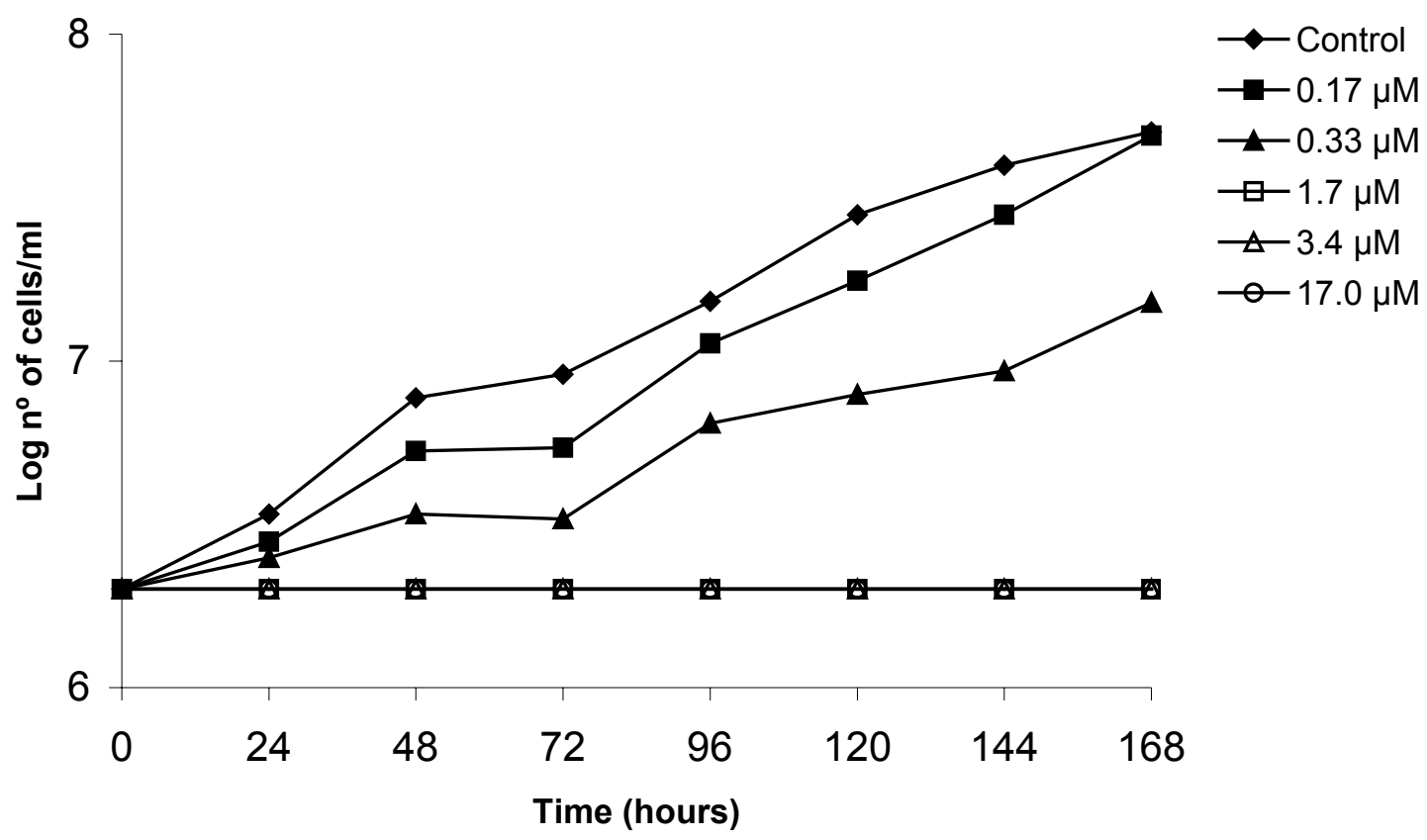

Figure 1. Dose response growth-curves of epimastigote forms of $T$. cruzi cultivated in presence of several concentrations of 5'-S-Methyltioadenosine.

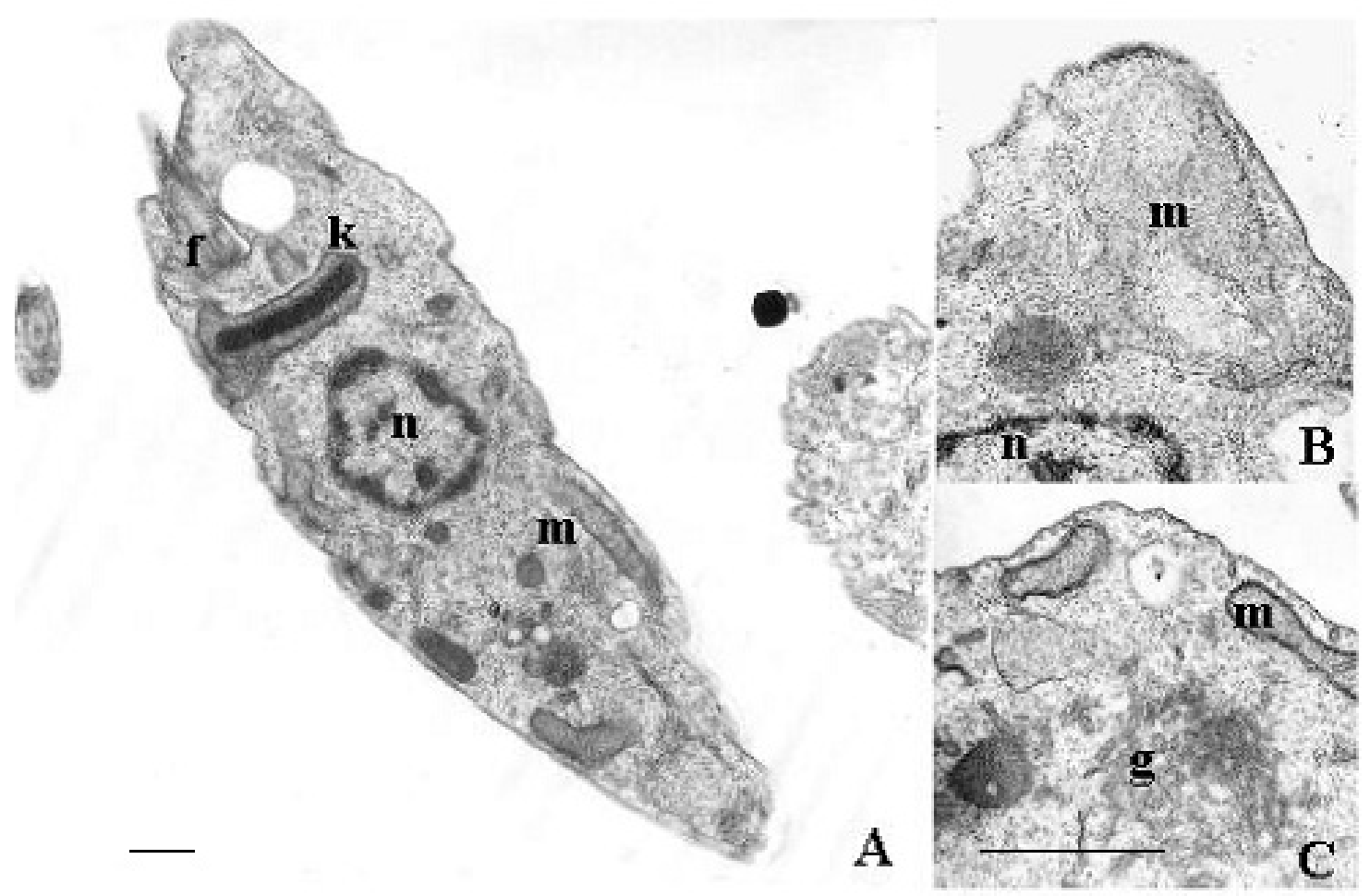

Figure 2. Transmission electron microscopy of Trypanosoma cruzi grown in LIT medium. (A) Untreated cell; (B and C) Cells treated with 5'-S-Methyltioadenosine $\mathrm{IC}_{50}$ of $\sim 0.236 \mu \mathrm{M}$ after 120 hours of culture - f, flagellum; k, kinetoplast; m, mitochondrion; n, nucleus; g, Golgi complex. Bars = $1 \mu \mathrm{m}$. 